\title{
Sector decomposition via computational geometry
}

\section{Toshiaki Kaneko*}

High Energy Accelerator Research Organization (KEK)

1-1 Oho, Tsukuba, Ibaraki 305-0801, Japan

E-mail: toshiaki.kanekodkek. ip

\section{Takahiro Ueda}

Graduate School of Pure and Applied Sciences, University of Tsukuba,

Tennodai, Tsukuba, Ibaraki 305-8577, Japan

E-mail: tuedaehet.ph.tsukuba.ac.jp

\begin{abstract}
A non-iterative method is presented for the factorization step of sector decomposition method, which separates infrared divergent part from loop integration. This method is based on a classification of asymptotic behavior of polynomials. The problem is converted to ones for convex body in Euclidean space. They are solved with algorithms developed in computational geometry. A test implementation shows that this method produces less number of decomposed sectors than usual iterative sector decompositions.
\end{abstract}

13th International Workshop on Advanced Computing and Analysis Techniques in Physics Research February 22-27, 2010

Jaipuri, India

\footnotetext{
${ }^{*}$ Speaker.
} 


\section{Introduction}

In the calculation of Feynman amplitude with massless particles, one has to regulate infrared divergences (IR), which cancel out among loop corrections and real emission processes. In perturbative QCD, this divergences are regulated by $D$-dimensional method. Divergent part is expressed as poles in terms of $\varepsilon=(4-D) / 2$. Sector decomposition method developed in Refs. [四, 口, 回, 团] are widely used for separating IR divergences.

As a simple example of separation of infrared divergences, let us consider the following onedimensional integral for $\varepsilon<0$ with a regular function $f$ such that $f(0)$ is a non-zero finite value:

$$
\begin{aligned}
I & =\int_{0}^{1} d x x^{-1-\varepsilon} f(x) \\
& =\int_{0}^{1} d x x^{-1-\varepsilon} f(0)+\int_{0}^{1} d x x^{-1-\varepsilon}(f(x)-f(0)) \\
& =-\frac{f(0)}{\varepsilon}+\int_{0}^{1} d x\left(x^{-\varepsilon} f^{\prime}(0)+\frac{1}{2} x^{1-\varepsilon} f^{\prime \prime}(0)+\ldots\right) .
\end{aligned}
$$

When $\varepsilon=0$, the factor $x^{-1-\varepsilon}$ produces a logarithmic divergence by the integration around $x \sim 0$. While for $\varepsilon<0$, the divergence is regularized and converted to a pole of $\varepsilon$. The coefficient of the pole is $f(0)$, which is the first term of the Taylor expansion of $f(x)$ around $x=0$. The rest of the integration becomes finite for $\varepsilon \rightarrow 0$. This example shows that, when the singular part of the integrand is factored out, one can separate divergent part as poles in terms of $\varepsilon$.

For the general loop integrations, it will also be possible to separate IR divergence, if the singular part is factored out. Although the factorization of a multi-variate polynomial is not a trivial problem, it is solved by the method of sector decomposition. This method shows that when the integration domain is properly decomposed into sectors and selecting good variables in each sector, the divergent part is factored out. This method provides at the same time a practical procedure to find such sectors and variables. Then we can obtain the coefficients of the poles of $\varepsilon$ and the finite part as a Laurent series in terms of $\varepsilon$.

Usual sector decomposition method divides the integration domain and find appropriate new variables by an iterative way. It was found that a simple iterative method may fall into an infinite

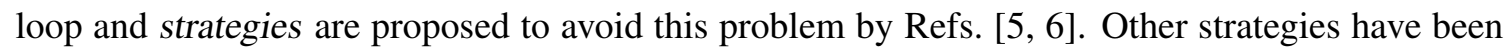

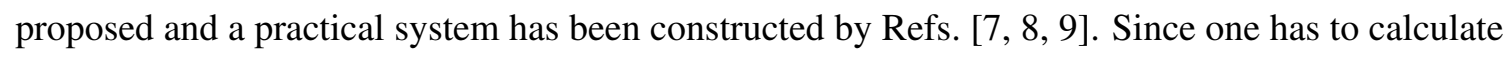
integration for each sector, a method is preferable when it produces less number of sectors.

We propose another method based on the classification of asymptotic behavior of polynomials around the origin. The problem is converted to one for convex bodies in Euclidean space and solved with algorithms developed in computational geometry. These are deterministic algorithms without iterations. This talk presents, based on Ref. [ए]], our basic ideas and show the results of this method.

\section{Sector decomposition}

The procedure of sector decomposition consists of the following steps:

1. Start from Feynman parameter representation of a loop integration. 
2. Primary sector decomposition:

The $\delta$-function is integrated and the integration domain is decomposed such that the singularities appear in the specific position in each sector.

3. Factorization of the integrand:

The sectors obtained in the previous step are decomposed into finer ones. New variables are found such that singular parts are factored out.

4. Separation of poles in terms of $\varepsilon$ :

The regular part of the integrand is expanded in terms of $\varepsilon$. The coefficients of Laurent series in terms of $\varepsilon$ are obtained.

5. Integration of coefficients:

The coefficients are now free from IR divergences. However, they will still include multidimensional integration. They will be integrated out by analytic or numerical methods.

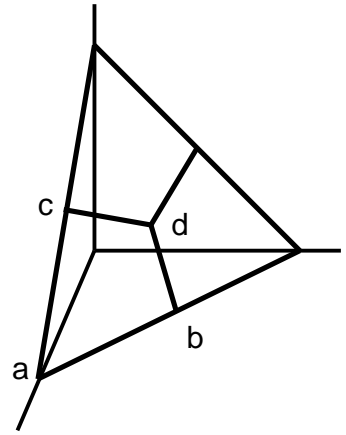

(a)

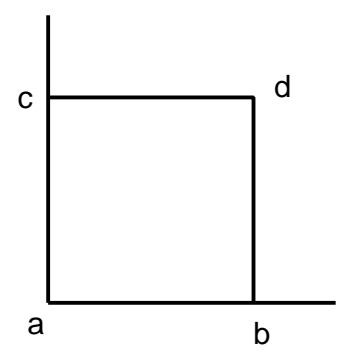

(b)

Figure 1: An example of primary sector decomposition in 3-dimensional case. Integration plane is divided into 3 sectors and sub-domain abcd in (a) is mapped to a square shown by (b).

Feynman parameter representation of a loop integration includes $\delta$-function which defines the $(N-1)$-dimensional hyperplane. The primary sector decomposition integrates over one of integration variables and decompose the integration domain into $N$ sectors. An example is shown by Fig. $\square$ for the 3-dimensional case. By the decomposition of the integration domain and selecting new variables, sectors become $(N-1)$-dimensional cubes. The original boundary of the integration domain is mapped to a part of the new boundary specified by conditions that some of new variables are 0 . The resulting expression of the integration for $l$-th sector becomes:

$$
G_{l}=\int_{0}^{1} d^{N-1} t t^{\nu-\mathbb{I}} \mathscr{U}_{l}^{\gamma}(t) \mathscr{F}_{l}^{\beta}(t), \quad t^{\nu-\mathbb{I}}=\prod_{j=0}^{N-1} t_{j}^{v_{j}-1},
$$

where $\mathscr{F}_{l}(t)$ and $\mathscr{U}_{l}(t)$ are polynomials of variables $t$. Parameter $v_{j}$ is integer determined by the power of propagators, and $\mathbb{I}$ is an $(N-1)$-dimensional vector whose elements are all 1 . Powers $\beta$ and $\gamma$ are functions of $\varepsilon$. If $\mathscr{F}_{l}(t)$ or $\mathscr{U}_{l}(t)$ become 0 at the boundary, and corresponding power $\beta$ or $\gamma$ becomes negative integer for $\varepsilon=0$, the integration diverges. 


\section{Geometric method}

We want to express polynomial $\mathscr{F}_{l}(t)$ in the form:

$$
\mathscr{F}_{l}(t(z))=C_{a} z^{b_{a}}\left(1+H_{a}(z)\right), \quad H_{a}(0)=0, \quad z^{b_{a}}=\prod_{j} z_{j}^{\left(b_{a}\right)_{j}}
$$

where $z$ is a set of new variable, $H_{a}(z)$ is a polynomial of $z, b_{a}$ is an integer vector and $C_{a}$ is a constant. In order to seek such an expression, we consider the following example taken from one-loop box integration:

$$
\mathscr{F}_{l}(t)=-s_{23} t_{2} t_{3}-s_{12} t_{1}-s_{4} t_{1} t_{3}=t_{2} t_{3}\left(-s_{23}-s_{12} \frac{t_{1}}{t_{2} t_{3}}-s_{4} \frac{t_{1}}{t_{2}}\right) .
$$

If terms $t_{1} / t_{2} t_{3}$ and $t_{1} / t_{2} \rightarrow 0$ when $t_{2} t_{3} \rightarrow 0$, the asymptotic behavior of $\mathscr{F}_{l}$ is determined by the term $-s_{23} t_{2} t_{3}$. The singular behavior of the integrand around the origin is determined by this term. This condition is satisfied by taking new variables $z$ defined by $t_{2}=z_{2}, t_{3}=z_{3}, t_{1}=z_{1} z_{2} z_{3}$. With the range of $z_{j}$ being limited to $(0,1)$, a sub-domain of the integration domain in $t$-space is obtained. This example shows that a term of a polynomial in some sub-domain will determine asymptotic behavior of the polynomial around the origin. We call this term dominant in this sub-domain.

In order to see dominant terms more closely around the origin, let us change variable to $y_{j}=$ $-\log t_{j}$ or $t_{j}=e^{-y_{j}}$. Monomial $t^{c}=t_{1}^{c_{1}} t_{2}^{c_{2}} \cdots t_{N-1}^{c_{N-1}}$ becomes $e^{-(c, y)}$, where $(c, y)=\sum c_{j} y_{j}$ is the inner product defined in $(N-1)$-dimensional Euclidean space. This shows that a monomial corresponds one-to-one to a integer vector $c$ in this Euclidean space (let us call this power vector). Let $Z^{\mathscr{F}_{l}}$ is the set of power vectors corresponding to the terms included in a polynomial $\mathscr{F}_{l}$. Polynomial $\mathscr{F}_{l}$ is expressed by:

$$
\mathscr{F}_{l}(t)=\sum_{c \in Z^{\mathscr{F}_{l}}} a_{c} t^{c}=\sum_{c \in Z^{\mathscr{F}_{l}}} a_{c} e^{-(c, y)}
$$

In order to find the dominant term, let us consider a limit $\lambda \rightarrow+\infty$ for $y_{j}=\lambda u_{j} \rightarrow+\infty$ with a fixed non-negative real vector $u$. A term $t^{b}=e^{-(b, y)}$ is dominant in this limit when $(b, y) \leq(c, y)$ for all $c \in Z^{\mathscr{F}_{l}}$. Conversely, let us fix vector $b$ and vary $u$ or $y$. The term with this power vector $b$ is dominant in the sub-domain defined by:

$$
\Delta_{b}^{\mathscr{F}_{l}}:=\left\{y \in \mathbb{R}_{\geq 0}^{N-1} \mid(c-b, y)>0, \forall c \in Z^{\mathscr{F}_{l}}\right\} .
$$

This sub-domain forms a convex polyhedral cone. The polynomial $\mathscr{F}_{l}$ is expressed as the following:

$$
\mathscr{F}_{l}(t(y))=\sum_{b \in Z^{\mathscr{F}_{l}}} \theta\left(y \in \Delta_{b}^{\mathscr{F}_{l}}\right) e^{-(b, y)}\left[a_{b}+\sum_{c \in Z^{\mathscr{F}_{l}}-\{b\}} a_{c} e^{-(c-b, y)}\right]
$$

For a vector $y \in \Delta_{b}^{\mathscr{F l}}$, factor $e^{-(c-b, y)} \rightarrow 0$ for $|y| \rightarrow \infty$, since $(c-b, y)>0$ holds for all $c$. This implies that the term $t^{b}$ is dominant in the sub-domain $\Delta_{b}^{\mathscr{F}_{l}}$. Thus, asymptotic behavior of $\mathscr{F}_{l}$ is classified in terms of these sub-domains $\left\{\Delta_{b}^{\mathscr{F} l}\right\}$.

When this method is applied also to $\mathscr{U}_{l}$, the singular parts are factored out from the integration. Let us return to our example Eq. (B.2) of one-loop box. In this case $\mathscr{U}_{l}$ is $1+t_{1}+t_{2}+t_{3}$ and this 


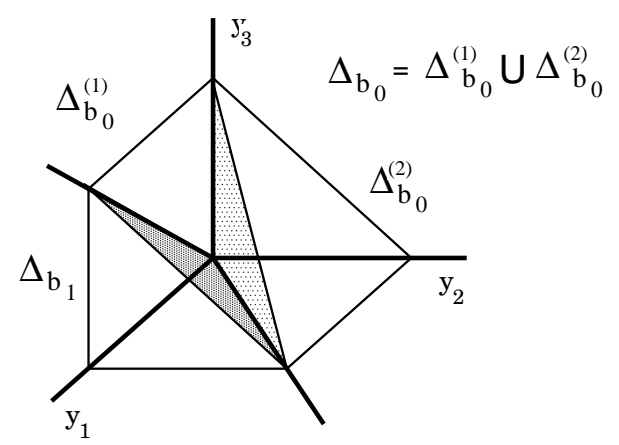

Figure 2: An example of geometric sector decomposition

expression shows that $\mathscr{U}_{l}$ does not produce IR singularity $\left(\mathscr{U}_{l} \rightarrow 1\right.$ for $\left.t_{i} \rightarrow 0\right)$. With a simple calculation, we obtain from Eq. (B.2):

$$
\begin{aligned}
t^{b_{0}} & =t_{1}, \quad t^{b_{1}}=t_{2} t_{3}, \quad t^{b_{2}}=t_{1} t_{3}, \\
Z^{\mathscr{F}_{l}} & =\left\{b_{0}=(1,0,0), b_{1}=(0,1,1), b_{2}=(1,0,1)\right\}, \\
\Delta_{b_{0}} & =\left\{x_{1}(1,1,0)+x_{2}(1,0,1)+x_{3}(0,1,0)+x_{4}(0,0,1) \mid x_{1}, x_{2}, x_{3}, x_{4} \geq 0\right\}, \\
\Delta_{b_{1}} & =\left\{x_{1}(1,0,0)+x_{2}(1,1,0)+x_{3}(1,0,1) \mid x_{1}, x_{2}, x_{3} \geq 0\right\}, \\
\Delta_{b_{2}} & =\emptyset .
\end{aligned}
$$

The sub-domains are shown in Fig. $\downarrow$. The fact that the last sub-domain is empty means that term $t_{1} t_{3}$ never becomes dominant. The second domain is a triangular cone for which variables $x$ take simple range of values. However, the first sub-domain includes four parameters in 3-dimensional space. When this sub-domain is divided further into two triangular cones, this redundancy disappears:

$$
\begin{aligned}
\Delta_{b_{0}} & =\Delta_{b_{0}}^{(1)} \cup \Delta_{b_{0}}^{(2)}, \\
\Delta_{b_{0}}^{(1)} & =\left\{x_{1}(1,1,0)+x_{2}(1,0,1)+x_{4}(0,0,1) \mid x_{1}, x_{2}, x_{4} \geq 0\right\}, \\
\Delta_{b_{0}}^{(2)} & =\left\{x_{1}(1,1,0)+x_{3}(0,1,0)+x_{4}(0,0,1) \mid x_{1}, x_{3}, x_{4} \geq 0\right\} .
\end{aligned}
$$

Now we subsequently change variable from $y_{j}$ to $x_{i}$ and then to $z_{i}=e^{-x_{i}}$. The integration domain for $z$ becomes 3-dimensional unit cube. Finally we obtain a sector decomposition as the following:

\begin{tabular}{lll}
\hline sector & \multicolumn{1}{c}{ variables } & \multicolumn{1}{c}{$Z^{\mathscr{F}_{l}}$} \\
\hline$\Delta_{b_{0}}^{(1)}$ & $t_{1}=z_{1} z_{2}, t_{2}=z_{1}, t_{3}=z_{2} z_{4}$ & $z_{1} z_{2}\left(-s_{12}-s_{23} z_{4}-s_{4} z_{2} z_{4}\right)$ \\
$\Delta_{b_{0}}^{(2)}$ & $t_{1}=z_{1}, t_{2}=z_{1} z_{3}, t_{3}=z_{4}$ & $z_{1}\left(-s_{12}-s_{23} z_{3} z_{4}-s_{4} z_{4}\right)$ \\
$\Delta_{b_{1}}$ & $t_{1}=z_{1} z_{2} z_{3}, t_{2}=z_{2}, t_{3}=z_{3}$ & $z_{2} z_{3}\left(-s_{12} z_{1}-s_{23}-s_{4} z_{1} z_{3}\right)$ \\
\hline
\end{tabular}

It is easy to calculate Jacobian, which is found to be a monomial of $z$.

For the general case, the decomposition of the integration domain in accordance with the classification of the asymptotic behavior, corresponding to Eqs. (B.4) and (B.6), becomes a problem in Euclidean geometry. It is not so hard to solve this when one uses convex hull algorithms developed 
in computational geometry. This decomposition is uniquely determined once a polynomial is given. It is independent of the choice of an algorithm. In order to obtain final representation of sector decomposition, corresponding to the previous table, it is necessary to triangulate convex polyhedral cones. This is performed with triangulation algorithms. The triangulation of convex polyhedral cones is not determined uniquely and the number of sectors will depend on the algorithm.

\section{Test implementation and conclusion}

We have made a test implementation of this method. Our convex hull algorithm is a modified one for convex polyhedral cones based on the algorithm described in Ref. [U] for polytopes. For triangulation of convex polyhedral cones, we have developed our own algorithm. The input to our program is given by the package described in Ref. [ए2] and output is passed to the same package for the separation of poles and integrations of coefficients. We have checked our program by comparison with another package qhull (Ref. [ए3]]) for the convex hull algorithm, calculating integration volume for the triangulation, and comparison of integrated values of several diagrams with references. The number of decomposed sectors are shown by Table $\mathbb{U}$.

Table 1: Comparison of number of sectors among different methods. Numbers in column " $\mathrm{H}$ " are cited from Ref. [四]. Columns "A", "B", "C", "S" and "X" indicate corresponding strategy described in [D] and [D]. As shown in $[\square]$, " $F$ " means that the sector decomposition fails and " $M$ " means that the memory overflow happened during the sector decomposition on a $8 \mathrm{~Gb}$ machine. The numbers with "*" are given by [44]]. "This method" indicates the number of sectors obtained by our method. "Exponential S.D." indicates the number of sectors before the triangulation.

\begin{tabular}{|l|lllll|l|l|l|}
\hline Diagram & A & B & C & S & X & H & $\begin{array}{l}\text { This } \\
\text { method }\end{array}$ & $\begin{array}{l}\text { Exponential } \\
\text { S.D. }\end{array}$ \\
\hline Bubble & 2 & 2 & 2 & $2^{*}$ & 2 & & 2 & 2 \\
Triangle & 3 & 3 & 3 & $3^{*}$ & 3 & & 3 & 3 \\
Box & 12 & 12 & 12 & 12 & 12 & & 12 & 8 \\
\hline Tbubble & 58 & 48 & 48 & $48^{*}$ & 48 & & 48 & 36 \\
Double box, $p_{i}^{2}=0$ & 775 & 586 & 586 & 362 & 293 & 282 & 266 & 106 \\
Double box, $p_{4}^{2} \neq 0$ & $543^{*}$ & $245^{*}$ & $245^{*}$ & $230^{*}$ & $192^{*}$ & 197 & 186 & 100 \\
Double box, $p_{i}^{2}=0$ & 1138 & 698 & 698 & $441^{*}$ & 395 & & 360 & 120 \\
$\quad$ nonplanar & & & & & & & & \\
D420 & 8898 & 564 & 564 & 180 & $\mathrm{~F}$ & & 168 & 100 \\
\hline 3 loop vertex (A8) & $4617^{*}$ & $1196^{*}$ & $1196^{*}$ & $871^{*}$ & $750^{*}$ & 684 & 684 & 240 \\
Triple box & $\mathrm{M}$ & 114256 & 114256 & 22657 & 10155 & & 6568 & 856 \\
\hline
\end{tabular}

We have proposed a factorization algorithm in sector decomposition. Our method is based on a classification of asymptotic behavior of polynomials. The problem is converted to one for convex bodies in Euclidean space. In order to find sector decomposition for a given integrand, we employed algorithms developed in computational geometry. This method is deterministic and never falls into an infinite loop. A test implementation shows that the number of decomposed sectors is less than iterated sector decomposition combined with several strategies. 
This work is supported in part by Ministry of Education, Science, and Culture, Japan under Grantin-Aid Nos. 20340063 and 21540286.

\section{References}

[1] T. Binoth and G. Heinrich, Nucl. Phys. B 585 (2000) 741 [arXiv:hep-ph/0004013].

[2] T. Binoth and G. Heinrich, Nucl. Phys. B 680 (2004) 375 [arXiv:hep-ph/0305234].

[3] T. Binoth and G. Heinrich, Nucl. Phys. B 693 (2004) 134 [arXiv:hep-ph/0402265].

[4] G. Heinrich, Int. J. Mod. Phys. A 23 (2008) 1457 [arXiv:0803.4177 [hep-ph]].

[5] C. Bogner and S. Weinzierl, Comput. Phys. Commun. 178 (2008) 596 [arXiv:0709.4092 [hep-ph]].

[6] C. Bogner and S. Weinzierl, Nucl. Phys. Proc. Suppl. 183 (2008) 256 [arXiv:0806.4307 [hep-ph]].

[7] A. V. Smirnov and M. N. Tentyukov, Comput. Phys. Commun. 180 (2009) 735 [arXiv:0807.4129 [hep-ph]].

[8] A. V. Smirnov and V. A. Smirnov, JHEP 0905 (2009) 004 [arXiv:0812.4700 [hep-ph]].

[9] A. V. Smirnov, V. A. Smirnov and M. Tentyukov, [arXiv:0912.0158 [hep-ph]].

[10] T. Kaneko and T. Ueda, [arXiv:0908.2897 [hep-ph]], to appear in Comput. Phys. Commun.

[11] H. Edelsbrunner. “Algorithms in Combinatorial Geometry,” Springer, 1987.

[12] T. Ueda and J. Fujimoto, PoS (ACAT08) (2008) 120 [arXiv:0902.2656 [hep-ph]].

[13] Program package qhull, http://www. qhull.org/.

[14] Private communication with A. V. Smirnov. 